\title{
A Brucellosis Febrile Syndrome Following Meningeal Involvement
}

\author{
Wali GY ${ }^{1}$, Al-Turkistani $\mathrm{H}^{1}$ and Qutub $\mathrm{MO}^{2 *}$ \\ ${ }^{1}$ Department of Internal Medicine, division of Infectious Diseases King Faisal Specialist Hospital and Research Centre, Jeddah, \\ Saudi Arabia \\ ${ }^{2}$ Department of Pathology and Laboratory Medicine, division of Microbiology, King Faisal Specialist Hospital and Research \\ Centre, Jeddah, Saudi Arabia
}

*Corresponding author: Qutub MO, Department of Pathology and Laboratory Medicine, Division of Microbiology, MBC\#J-10, DPLM, King Faisal Specialist Hospital \& Research Centre, P.O. Box 40047, Jeddah-21499, Saudi Arabia, Fax: 012-6698620, Tel: 012-6677777 (Ext.63086), E-mail: Qutub.mohammed@kfshrc.edu.sa

Citation: Wali GY, Al-Turkistani H, Qutub MO (2018) A Brucellosis Febrile Syndrome Following Meningeal Involvement. SAJ Case Rep: 5:104

Article history: Received: 15 January 2018, Accepted: 15 February 2018, Published: 19 February 2018

\begin{abstract}
Neurobrucellosis (NB) is an uncommon presentation even in countries where brucellosis is endemic; only a few cases have been reported. We report a case of a 23-year-old female who presented with typical symptoms of brucellosis including fever, night sweets, anorexia, back pain and weight loss. Her blood culture grew Brucella melitensis and the serology tested strongly positive for brucella antibodies. The patient was treated initially with doxycycline and rifampicin and then was put on doxycycline and trimethoprim-sulfamethaxazole (TMP-SMX) due to drug-induced hepatitis secondary to rifampicin. Later on, she got pregnant and was lost to follow up. She presented one week post-partum with fever and severe frontal headaches in which a contrast-enhanced magnetic resonance imaging (MRI) brain was obtained and revealed diffuse meningeal enhancement involving the anterior and posterior fossa consistent with pachymeningeal enhancement. The patient was treated with doxycycline, gentamycin and ceftriaxone. Follow up MRI showed marked improvement of the meningeal enhancement that was previously observed.
\end{abstract}

Keywords: Neurobracellosis; Brucella

\section{Introduction}

Brucellosis is one of the zoonotic endemic diseases in Saudi Arabia. It is transmitted to humans through ingestion of unpasteurized milk, milk products or by contact with infected animals or animal discharges. Rarely, transmission has occurred via sex or to babies via placenta or breast milk [1]. On the other hand, laboratory-acquired brucellosis has continued to occur and reported in several cases [2]. There are many species of Brucella and the most important types causing human disease are: Brucella melitensis, Brucella abortus, Brucella suis and Brucella canis. Clinical studies showed that Fever followed by osteoarticular involvement is the commonest features of brucellosis [3]. However, Neurobrucellosis (NB) occur in 2-5\% of patients [4,5]. Patients can present with acute or chronic neurological manifestations such as meningitis, transverse myelitis, brain abscess, cranial nerve involvement, seizures and depression. Meningitis has been referred to as the most frequent presentation of NB occurring in at least the 50\% of cases [4,6]. Meningitis is most often acute, but subacute and chronic presentations are not rare and may lead to disseminated encephalomyelitis with diffuse central nervous system (CNS) demyelinization [4]. Here, we report a case of a healthy female microbiologist that got infected by inhalation during handling a positive sample in the lab. She failed her treatment due to side effects and poor compliance and had a complicated course of the disease with brucella meningitis based on her symptoms, positive blood cultures, brucella serology and significant MRI findings.

\section{Case report}

The patient is a 23-year-old microbiology technician woman, she presented to the King Faisal Specialist Hospital and Research Centre with a week history of high degree continuous fever, night sweats, anorexia, back pain, fatigability and weight loss. She had no animal contact or raw milk ingestion. The patient lives in Jeddah; she reports a history of handling a positive brucella sample in the lab during her duty one month prior to her illness. 
Upon examination, patient's temperature was $37.7^{\circ} \mathrm{C}$, blood pressure $110 / 75 \mathrm{~mm} \mathrm{Hg}$, heart rate 96 beats per minute. There was no lymphadenopathy, cardiac murmurs, abdominal tenderness or hepatosplenomegaly. The findings of the rest of the examination were either negative or within normal range.

Laboratory investigations revealed that haemoglobin was $133 \mathrm{~g} / \mathrm{L}$, platelets $170 \mathrm{x} 10^{9} / \mathrm{L}$, and WBC 3.07x10 $/ \mathrm{L}$. Other biochemical parameters including renal and liver functions tests were within normal limits. Chest X-ray was normal. Blood culture was positive for gram negative coccobacilli, which was identified as Brucella melitensis by a combination of the culture and nucleic acid sequencing of the $16 \mathrm{~S}$ ribosomal RNA (rRNA) gene for the speciation. Brucella serology was done by the microdilution version of the standard agglutination test (SAT) by using a stained Brucella abortus antigen suspension to identify and quantitate specific antibodies in serum, and was positive for brucella total antibodies (IgM and IgG) with a titer of (1:2560). Then IgG was determined by treatment of serum with 2-mercaptoethanol (2-ME) which destroys IgM agglutinating antibodies and was positive a titer of (1:160). Difference between the 2-ME titer and a total antibody titer gives an indirect measure of the level of IgM antibody.

We started her on doxycycline and rifampicin. A week later, she developed drug-induced hepatitis secondary to rifampicin, this was manifested by elevation of her liver enzymes and bilirubin; therefore, patient's regimen was changed to doxycycline and TMPSMX. Around 2 weeks later, the patient got pregnant and stopped taking her medications despite minimal improvement of her symptoms; she also missed all her follow up appointments with the infection disease clinic.

She presented to the hospital immediately post-partum (which is 10 months after discontinuation of her regimen) with high-grade fever and severe frontal headaches. She had mentioned that these symptoms started 2 weeks before presenting to our hospital; two sets of blood culture were collected and again reported positive for gram negative coccobacilli and brucella serology in blood was positive for brucella total antibodies (1:320), and for IgG antibodies (1:320).

An echocardiogram, CT abdomen, pelvis and spine were ordered and reported normal. A MRI of the brain revealed diffuse meningeal enhancement involving the anterior and posterior fossa, which also involved the cervical spine area consistent with pachymeningeal enhancement (Figure 1). Lumber puncture yielded a clear cerebrospinal fluid (CSF) containing 10 white blood cells $/ \mathrm{mm}^{3}$ with $80 \%$ lymphocytes. The CSF glucose was $3 \mathrm{mmol} / \mathrm{L}$ and CSF protein was $0.341 \mathrm{~g} / \mathrm{L}$, No microorganisms were observed in a gram stained CSF smear or on culture and serology test on CSF was negative.

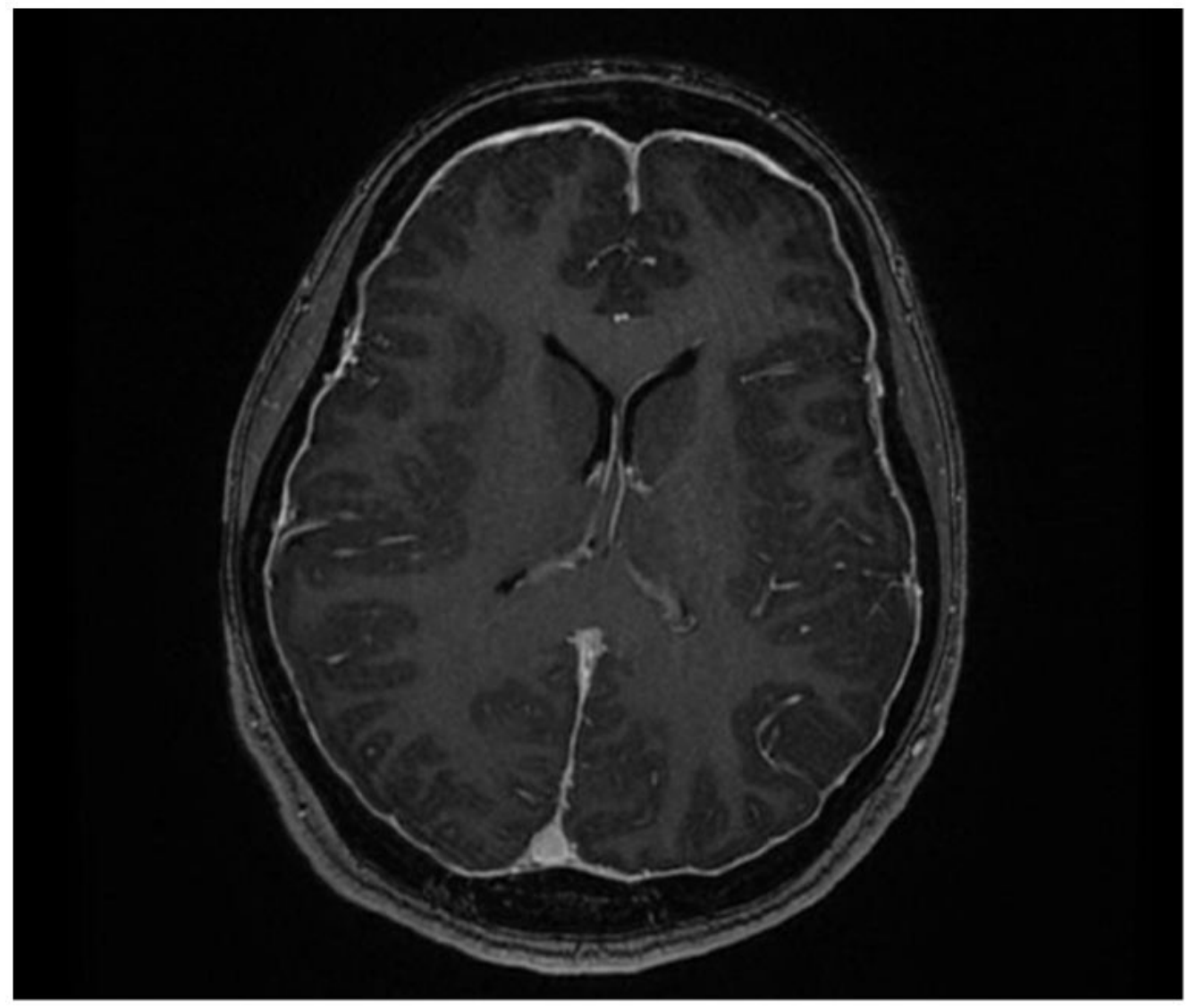

Figure 1: MRI of the brain showing diffuse meningeal enhancement involving the anterior and posterior fossa consistent with pachymeningeal enhancement

Our patient was treated with doxycycline, gentamycin and ceftriaxone. Follow up MRI was requested a month later and showed marked improvement of the meningeal enhancement that was previously observed (Figure 2). She was subsequently treated for one-year with doxycycline, TMP-SMX and ciprofloxacin and has made full recovery from all her symptoms. 


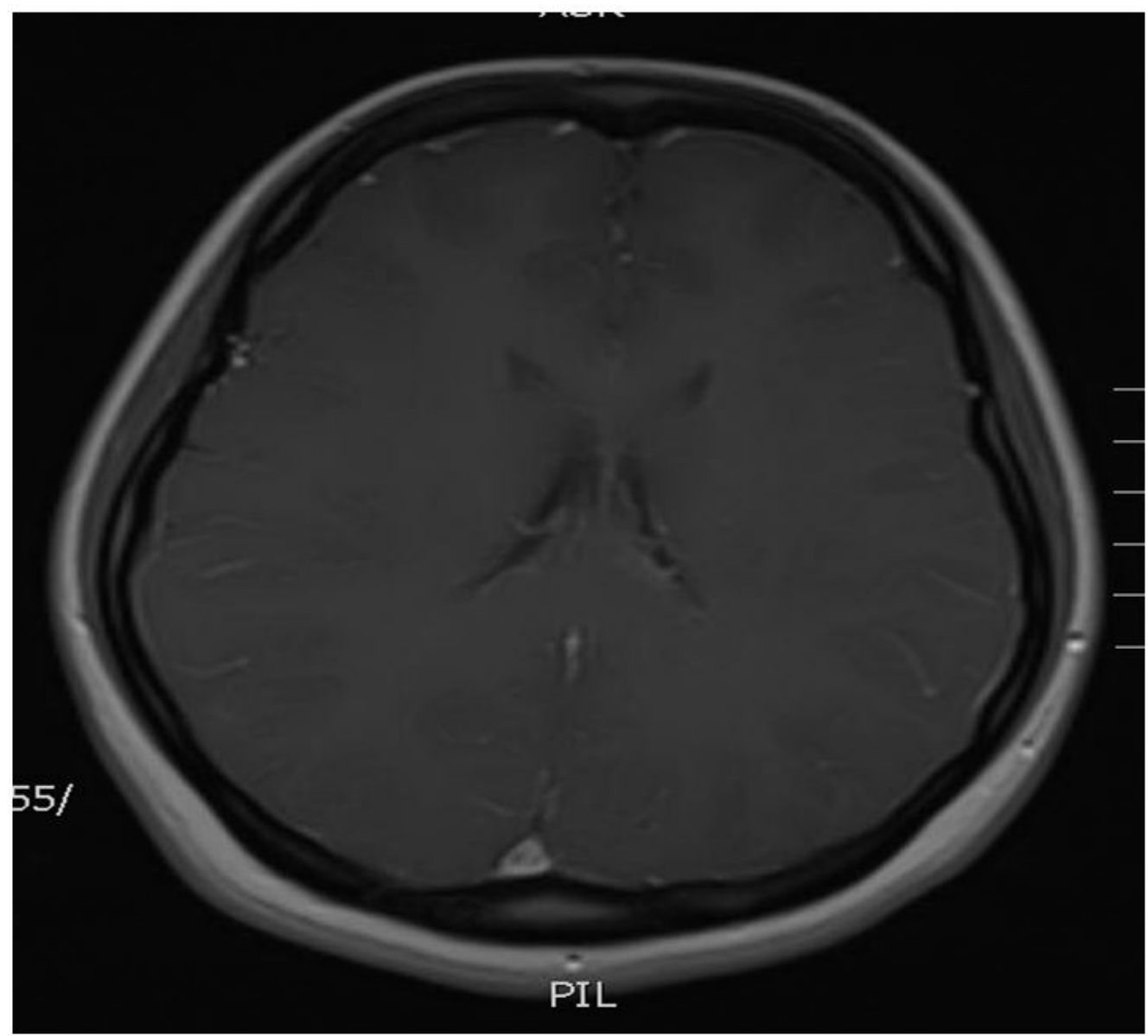

Figure 2: MRI of the brain showing significant improvement of the meningeal enhancement after treatment

\section{Discussion}

Brucellosis is one of the most important endemic infections in our region. The potential hazard of Brucella spp. to laboratory personal has long been recognized, through the years, brucellosis has remained the most commonly reported laboratory-associated bacterial infection [7-11]. The World Health Organization classifies brucellosis as Risk Group III pathogen, meaning that it poses a high risk to person but a low risk to the community. Aerosol transmission generated accidentally or during microbiologic techniques from contaminated materials is the most frequently reported cause of disease acquisition in the laboratory [8-11]. In our case, the patient is a medical technologist working in microbiology laboratory and was handling a positive culture that was misinterpreted as gram variable by gram stain. As a result, all the processes of this positive culture were handled in open bench and that led to the aerosol transmission of this infection.

Culture of cerebrospinal fluid is often negative in patients with NB especially when a meningoencephalitis disease is present. In our patient, the evidence of systemic disease, clinical history, the cerebrospinal fluid features, and the response to therapy all strongly favored the diagnosis of NB. There are no specific MRI findings for NB; pachymeningeal enhancement also known as duraarachnoid enhancement can be seen in infections, intracranial tumors, intracranial hypotension, neurosarcoidosis and rheumatoid arthritis [12].

Neurobrucellosis has a wide array of clinical presentations. Our patient presented only with headaches and without any peripheral neurological signs. This has been consistent with previous cases [13].

The management of neurobrucellosis requires special attention since the illness is potentially life threatening and conventional antibiotics used in management of CNS infections like pencillin, chloramphenicol, cephalosporins and carbapenems are ineffective. Most experts recommend the combination of two or three drugs which cross the blood-brain-CSF barrier (such as doxycycline, rifampicin, trimethoprim-sulfamethoxazole, ciprofloxacin, ceftriaxone) [14,15]. Treatment needs to be individualized according to signs and symptoms but should generally be prolonged, it varies from 1 to 19 months [14,15]. We recommend treating these patients for at least one year.

We cannot overemphasize the importance of completion of treatment of this disease. Our patient stopped her treatment prematurely and that has led to a relapse in the most unusual location. Appropriate treatment for the intended duration prevents such rare complications. 


\section{Conclusion}

The main outcome of this case study is to bring to the attention of physicians the unusual manifestations and difficulty of diagnosing NB; this rare entity should be considered in microbiology technicians, with fever and unexplained neurological manifestations.

\section{References}

1. Tuon FF, Gondolfo RB, Cerchiari N (2017) Human-to-human transmission of Brucella - a systematic review. Trop Med Int Health 22: 539-46.

2. Memish Z, Mah M (2001) Brucellosis in laboratory workers at a Saudi Arabian hospital. Am J Infect Control 29: 48-52.

3. Turan H, Serefhanoglu K, Karadeli E, Togan T, Arslan H (2011) Osteoarticular involvement among 202 brucellosis cases identified in Central Anatolia region of Turkey. Intern Med 50: 421-8.

4. McLean DR, Russell N, Khan MY (1992) Neurobrucellosis; clinical and therapeutic features. Clin Infect Dis 15: 582-90.

5. Mousa AR, Koshy TS, Araj GF, Marafie AA, Muhtaseb SA, et al. (1986) Brucella meningitis: presentation, diagnosis and treatment: a prospective study of ten cases. Q J Med 60: 873-85.

6. Guven T, Ugurluk K, Ergonul O, Celikbas AK, Gok SE, et al. (2013) Neurobrucellosis: Clinical and Diagnostic features. Clin Infect Dis 56: 1407-12.

7. Pike RM (1978) Past and present hazards of working with infectious hazards. Arch Pathol Lab Med 102: 333-6.

8. Gruner E, Bernasconi E, Galeazzi R, Buhl D, Heinzle R, et al. (1994) Brucellosis: an occupational hazard for medical personnel. Report of five cases. Infection 22: 33-6.

9. Olle-Goig JE, Canola-Soler J (1987) An outbreak of Brucella melitensis infection by airborne transmission among laboratory workers. Am J Public Health 77: $335-8$.

10. Grammont-Cupillard M, Berthet-Badetti L, Dellamonica P (1996) Brucellosis from sniffing bacteriological cultures. Lancet 348: 1733-4.

11. Flori P, Mastrandrea S, Rappelli P, Cappuccinalli P (2000) Brucella abortus infection acquired in microbiology laboratories. J Clin Microbiol 38: 2005-6.

12. Tosaka M, Sato N, Fujimaki H, Takahashi A, Saito N (2005) Wave-like appearance of diffuse pachymeningeal enhancement associated with intracranial hypotension. Neuroradiology 47: 362-7.

13. Gul HC, Erdem H, Bek S. (2009) Overview of neurobrucellosis: a pooled analysis of 187 cases. Int J Infect Dis 13: 339-43.

14. Kizilkilic O, Turunc T, Yildirim T, Demiroglu YZ, Hurcan C, et al. (2005) Successful medical treatment of intracranial abscess caused by Brucella spp. J Infect 51: 77-80.

15. Karsen H, Tekin Koruk S, Duygu F, Yapici K, Kati M. (2012) Review of 17 cases of neurobrucellosis: clinical manifestations, diagnosis, and management. Arch Iran Med 15: 491-4. 